\title{
Ultra-stable microwave generation with a diode-pumped solid-state laser in the $1.5-\mu \mathrm{m}$ range
}

\author{
Vladimir Dolgovskiy $\cdot$ Stéphane Schilt $\cdot$ Nikola Bucalovic • \\ Gianni Di Domenico · Serge Grop • Benoît Dubois • \\ Vincent Giordano • Thomas Südmeyer
}

Received: 4 June 2013/ Accepted: 29 November 2013/Published online: 18 December 2013

(c) Springer-Verlag Berlin Heidelberg 2013

\begin{abstract}
We demonstrate the first ultra-stable microwave generation based on a $1.5-\mu \mathrm{m}$ diode-pumped solidstate laser (DPSSL) frequency comb. Our system relies on optical-to-microwave frequency division from a planarwaveguide external cavity laser referenced to an ultra-stable Fabry-Perot cavity. The evaluation of the microwave signal at $\sim 10 \mathrm{GHz}$ uses the transportable ultralow-instability signal source ULISS ${ }^{\circledR}$, which employs a cryo-cooled sapphire oscillator. With the DPSSL comb, we measured $-125 \mathrm{dBc} / \mathrm{Hz}$ phase noise at $1 \mathrm{kHz}$ offset frequency, likely limited by the photo-detection shot-noise or by the noise floor of the reference cryo-cooled sapphire oscillator. For comparison, we also generated low-noise microwave using a commercial Er:fiber comb stabilized in similar conditions and observed $>20 \mathrm{~dB}$ lower phase noise in the microwave generated from the DPSSL comb. Our results confirm the high potential of the DPSSL technology for low-noise comb applications.
\end{abstract}

\section{Introduction}

Low-noise microwave signals are of prime importance for numerous areas such as high-resolution radar systems, timing

\footnotetext{
V. Dolgovskiy $\cdot$ S. Schilt $(\bowtie) \cdot$ N. Bucalovic $\cdot$

G. Di Domenico · T. Südmeyer

Laboratoire Temps-Fréquence, Institut de physique, Université de Neuchâtel, Avenue de Bellevaux 51, 2000 Neuchâtel, Switzerland

e-mail: stephane.schilt@unine.ch

S. Grop · B. Dubois · V. Giordano

Time and Frequency Department, UMR 6174 CNRS,

FEMTO-ST Institute, UFC, ENSMM, Chemin de 1'Epitaphe 26, 25030 Besançon Cedex, France
}

distribution and synchronization, very-long baseline interferometry, space-borne gravitational wave detectors [1] or performance improvements of primary frequency standards based on cold cesium atoms [2,3]. Until recent years, the microwave signals providing the lowest phase noise and the highest frequency stability at short timescale $(<100 \mathrm{~s})$ were obtained from cryogenically cooled sapphire whispering gallery mode oscillators (CSO) $[4,5]$. Following the advent of optical frequency combs [6], the use of an ultra-stable laser providing an extremely stable optical frequency at short timescales, combined with a frequency comb acting as an optical-to-microwave frequency divider, constitutes an attractive alternative for ultra-low phase noise microwave generation, as first proposed by Ye et al. [7]. Optical references based on lasers stabilized to high-finesse, vibration-insensitive, ultra-low thermal expansion (ULE) glass Fabry-Perot cavities have reached unprecedented short-term fractional frequency stability in the few $10^{-16}$ range at $1 \mathrm{~s}$ as first demonstrated by the seminal work of Young et al. [8] and by other works later [9, 10]. The fundamental limit of ULE cavities arising from thermal noise in the dielectric coating of the mirrors has recently been overcome by the cryogenic single crystal silicon cavity developed by Kessler et al. [11], leading to fractional frequency stability lower than $10^{-16}$ at $1 \mathrm{~s}$.

Microwave generated through frequency division using a Ti:sapphire ( $\mathrm{Ti}: \mathrm{Sa}$ ) comb has been demonstrated at the level of $8 \times 10^{-16}$ at $1 \mathrm{~s}$ [12], but this type of combs suffers from its complexity, high cost and inefficient pumping. Alternatively, fiber-based optical frequency combs showing better repeatability and reliability have been regularly used for ultra-stable microwave generation [2, 13-15]. As examples, a fractional frequency stability in the low $10^{-15}$ at $1 \mathrm{~s}$ has been obtained for the microwave generated with a $1.55-\mu \mathrm{m}$ Er:fiber frequency comb [13]. However, noise suppression in fiber combs generally remains more 
challenging and usually requires higher feedback bandwidth such as obtained using an intra-cavity electro-optic modulator (EOM) [16]. The resulting possibility to use the pump diode current to stabilize the output power of the femtosecond laser has led to the lowest residual phase noise of $-120 \mathrm{dBc} / \mathrm{Hz}$ at $1 \mathrm{~Hz}$ offset from an $11.55-\mathrm{GHz}$ carrier demonstrated for the optical-to-microwave frequency division process when the microwaves generated from two Er:fiber combs stabilized to a common optical reference were compared [15]. Operation of a mode-locked Er:fiber laser with significantly lower noise has been demonstrated by Kim et al. [17], achieved in particular by carefully tuning the net cavity dispersion close to zero, leading to sub-100 as timing jitter of the optical pulse train. With these noise properties, 10-GHz microwave with phase noise at the level of $-140 \mathrm{dBc} / \mathrm{Hz}$ could be obtained from a free-running Er:fiber laser, but this estimation does neither account for the contribution of the carrier envelope offset (CEO) noise, nor for the photo-detection shot-noise arising from the relatively low repetition rate $(\sim 78 \mathrm{MHz})$ of this Er:fiber laser. In this sense, the multiplication of the comb repetition rate was shown as an efficient mean to improve the microwave phase noise by counteracting the lower pulse rate typically encountered in Er:fiber combs. Such a method was first proposed by Diddams et al. [18] using a filtering FabryPerot cavity and was later on implemented as a cascaded, unbalanced Mach-Zehnder fiber-based interferometer by Haboucha et al. [19], then by Jiang et al. [20].

A promising alternative to fiber combs are mode-locked diode-pumped solid-state lasers (DPSSLs). Similar to Ti:Sa lasers, they operate with high-Q cavities and moderate intra-cavity nonlinearities, resulting in a low fundamental quantum noise limit and a low-noise free-running CEO beat signal. This has been confirmed by the record narrow freerunning CEO linewidth of $<4 \mathrm{kHz}$ achieved in the $1.55-\mu \mathrm{m}$ spectral region from an Er:Yb:glass laser oscillator (ERGO) mode-locked with a semiconductor saturable absorber mirror (SESAM) [21]. Full stabilization of the ERGO comb was achieved with a residual integrated phase noise of the CEO beat of 0.72 rad rms obtained with a feedback bandwidth of only $5 \mathrm{kHz}$. These properties make the ERGO comb attractive for ultra-low-noise microwave generation, because the simplest possible scheme where one line of the self-referenced comb is phase-locked to the ultra-laser can be considered without the need for CEO subtraction as often implemented with Er:fiber combs to remove the higher contribution of the CEO frequency noise [13].

The first use of a DPSSL comb for low-noise microwave generation was demonstrated using an $\mathrm{Yb}: \mathrm{KYW}$ femtosecond laser [22]. A fractional frequency stability of $6 \times 10^{-15}$ was achieved at $1 \mathrm{~s}$ in comparison with the microwave signal generated from a Ti:Sa comb. Very recently, during the revision of the present paper, we have become aware of an improvement of these results to $2.5 \times 10^{-15}$ at $1 \mathrm{~s}$ [23], obtained by generating the microwave signal directly from the output of the $\mathrm{Yb}: \mathrm{KYW}$ laser rather than from the output of the nonlinear fiber used for supercontinuum generation. Here, we demonstrate the first ultra-stable microwave generation via optical frequency division using a SESAM-mode-locked DPSSL in the $1.5-\mu \mathrm{m}$ region, and we compare its performances (in terms of fractional frequency stability and phase noise) with those obtained in a similar setup using a commercial Er:fiber comb. A core element of our system is a planarwaveguide external cavity laser (PW-ECL) stabilized to a ULE Fabry-Perot cavity using the Pound-Drever-Hall (PDH) technique [24]. PW-ECLs are based on a relatively new semiconductor laser technology with low phase noise [25]. PW-ECLs are a promising alternative to fiber lasers or external cavity diode lasers (ECDL) traditionally used for the realization of robust ultra-stable optical frequency standards, because they can be cost-efficiently produced, and are compact and wavelength-agile. In the first demonstration of a stabilized PW-ECL reported by Numata et al. [26], a fractional frequency stability at the $10^{-13}$ level was obtained by stabilization to the $\mathrm{P}(16)$ acetylene $\left({ }^{13} \mathrm{C}_{2} \mathrm{H}_{2}\right)$ ro-vibrational transition at $1,542.383 \mathrm{~nm}$. This result was later on overcome by Clivati et al. [27], who stabilized a PW-ECL to a high-finesse Fabry-Perot cavity with a resulting residual fractional frequency stability of $2 \times 10^{-14}$, comparable with a distributed Bragg reflector fiber laser stabilized to the same cavity. Very recently, a fractional frequency stability below $10^{-15}$ has been measured at integration times ranging from 0.1 to $6 \mathrm{~s}$ for the beat signal between a $1.54-\mu \mathrm{m}$ PW-ECL and a $1.06-\mu \mathrm{m}$ Yb-doped fiber laser, both stabilized to high-finesse ULE cavities, using a femtosecond fiber comb to bridge the frequency gap between the two lasers [28].

We evaluated the microwave signal generated in our experimental setup by comparison with the transportable ultra-low instability signal source (ULISS) [29], based on a whispering gallery mode in a high-quality factor cryocooled sapphire resonator. This oscillator was developed at Femto-ST in Besançon and moved to Laboratoire TempsFréquence in Neuchâtel for a measurement campaign. ULISS offers a relative frequency stability (Allan deviation) better than $3 \times 10^{-15}$ at short term $(\tau<300 \mathrm{~s})$ and still better than $5 \times 10^{-15}$ over 1 day, which makes it very effective for the evaluation of microwave signals generated from optical frequency standards.

\section{Ultra-stable laser}

In our experiment, we used a commercial PW-ECL module (ORION from Redfern Integrated Optics Inc.). The 
fiber-coupled laser module contains a butterfly packaged PW-ECL with thermo-electrical control, a laser driver and a control interface. The laser has a preset center frequency of $192.5 \mathrm{THz}$, corresponding to the ITU-25 channel of the dense wavelength division-multiplexing (DWDM) grid of the international telecommunication union (ITU). It can be frequency-tuned over several gigahertz either via its injection current or by changing the diode temperature. We stabilized the laser to a Fabry-Perot cavity with both spacer and mirror substrates made out of ULE glass (ATFilms). The horizontally mounted $10-\mathrm{cm}$-long cavity has a measured finesse of $\sim 180,000$ [30] and a free-spectral range (FSR) of $\sim 1.5 \mathrm{GHz}$. We placed the cavity in a vacuum chamber at $4 \times 10^{-8}$ mbar residual pressure within a twolayer thermal enclosure made of a temperature-controlled external aluminum screen and an embedded internal copper layer for thermal insulation and temperature homogenization. The cavity is maintained at a temperature of $22.6^{\circ} \mathrm{C}$, corresponding to the measured turning point of its thermal expansion coefficient. We determined the static response of the PW-ECL frequency to a change of the injection current from the heterodyne beat between the laser and an optical frequency comb stabilized to an H-maser. With a tuning coefficient of $56 \mathrm{MHz} / \mathrm{mA}$, the laser frequency can be scanned with the injection current by three FSRs of the ULE cavity ( $\sim 27 \mathrm{~mA} / \mathrm{FSR})$, limited by the laser threshold ( $\sim 10 \mathrm{~mA})$ on one side and by the maximum operating current $(110 \mathrm{~mA})$ on the other side.

The PW-ECL is attractive for its low phase noise. We measured the frequency noise power spectral density (PSD) of the free-running PW-ECL from the heterodyne beat with an ultra-narrow linewidth laser, obtained by PDH stabilization of an ECDL to the ULE cavity. The frequency noise of the beat signal, which represents the frequency noise of the PW-ECL as a result of the negligible contribution of the cavity-stabilized ECDL, was measured using an RF frequency discriminator (Miteq FMDM 21.4/2-4) [31] and a fast Fourier transform (FFT) spectrum analyzer. Figure 1 displays the measured noise spectrum. The noise contributing to the laser linewidth extents up to Fourier frequencies of $2-3 \mathrm{kHz}$ only, according to the $\beta$-separation line concept introduced by Di Domenico et al. [32]. This is slightly lower than observed in the similar PW-ECL of Numata et al. [26] and Clivati et al. [27], where the crossing point with the $\beta$-separation line was instead in the range of $5-10 \mathrm{kHz}$. The corresponding linewidth of our PW-ECL, calculated from the frequency noise PSD using the simple approximation proposed in Ref. [32], amounts to $<10 \mathrm{kHz}$ (for an observation time of $0.1 \mathrm{~s}$ ). While the PW-ECL has a low frequency noise, a direct tight lock to the ULE cavity by feedback to the laser injection current was prevented by the low frequency modulation bandwidth of this laser. Figure 2 shows the frequency modulation

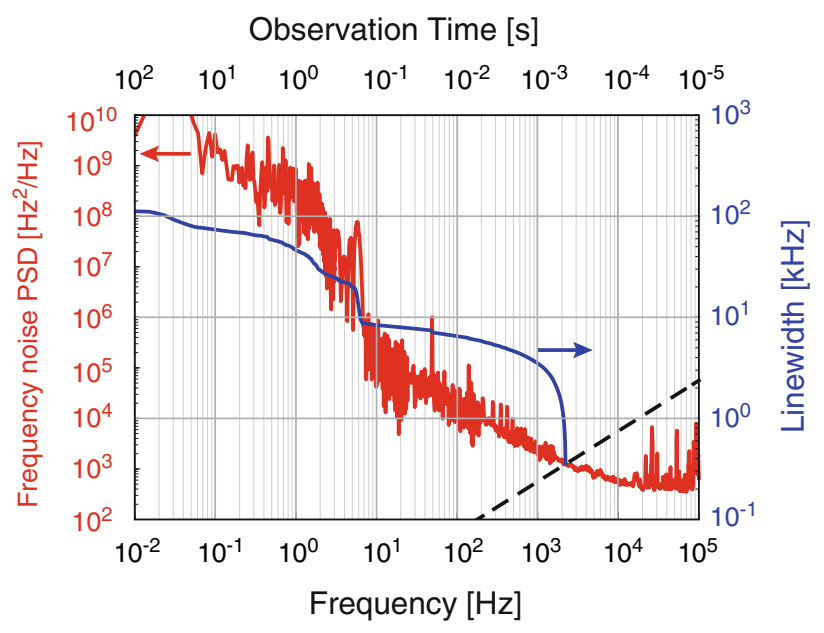

Fig. 1 (Color online) Frequency noise properties of the free-running PW-ECL. Left scale: frequency noise PSD. The $\beta$-separation line that is relevant for the determination of the linewidth [32] is also shown (dashed line). Right scale: linewidth (FWHM) of the free-running PW-ECL calculated from the frequency noise spectrum [32] as a function of the observation time (upper axis, increasing to left)

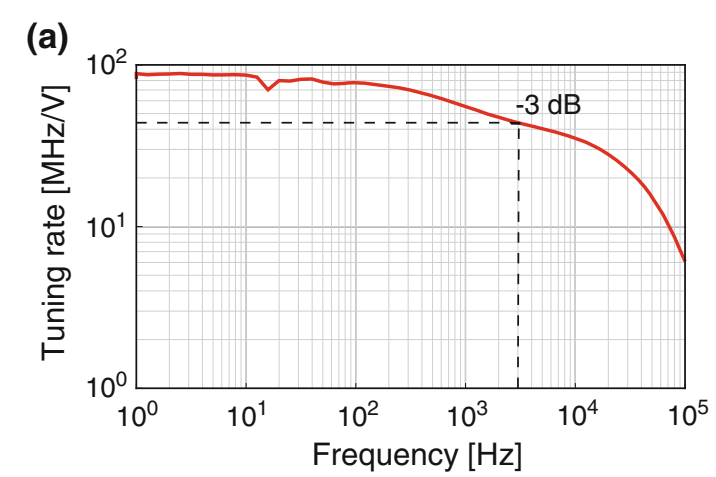

(b)

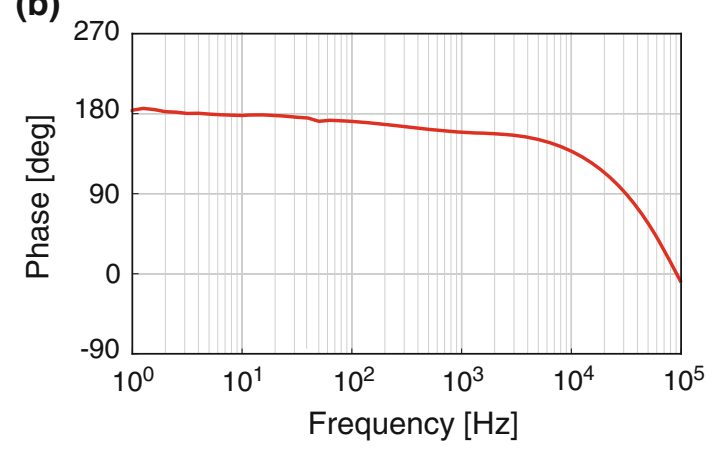

Fig. 2 (Color online) Transfer functions in amplitude (a) and phase (b) of the PW-ECL frequency for injection current modulation applied via the driver input voltage. The $-3 \mathrm{~dB}$ bandwidth (in amplitude) is $\sim 3 \mathrm{kHz}$

response (in amplitude and phase) of the laser measured as a function of the injection current modulation frequency. The measurement was performed by demodulating the heterodyne beat-note signal between the PW-ECL and a 
frequency comb referenced to an H-maser, using a frequency discriminator [31] and a lock-in detection. The 3-dB bandwidth (in amplitude) of $\sim 3 \mathrm{kHz}$ makes difficult to achieve a very tight lock of the laser to the cavity with feedback applied only to the laser injection current. This result is compatible with the observation of Numata et al. [26] and Clivati et al. [27] obtained with a similar laser module. However, this bandwidth limitation is believed to result from the internal laser driver of the ORION module and not from the laser itself. A much broader modulation bandwidth of many hundreds kilohertz has been shown for a PW-ECL driven by an external current source [28].

Therefore, a 40-MHz acousto-optic modulator (AOM, model FCM-40.8E5C from IntraAction Corp.) was used to achieve a higher feedback bandwidth (up to $100 \mathrm{kHz}$ ). Fast corrections were applied to the frequency modulation (FM) input of the AOM driver (DE-401 M) after amplification in a fast proportional-integral-derivative (PID) controller (FALC110 from Toptica). To prevent a drift of the AOM driving frequency that would result from the relative frequency drift between the free-running PW-ECL and the cavity resonance, slow corrections were applied to the laser current. This maintains the AOM driving frequency at its nominal value of $40 \mathrm{MHz}$, where the AOM diffraction efficiency is maximized. This minimizes power-to-frequency noise conversion that may result from a change of the optical power impinging on the cavity.

An electro-optical modulator (EOM, model PM-150005 from JDSU) was used to generate $30-\mathrm{MHz}$ FM sidebands in the laser spectrum for the PDH stabilization. The implemented laser stabilization scheme is depicted in Fig. 3. The laser power at the input of the ULE cavity was kept low $(\sim 50 \mu \mathrm{W})$ to minimize power-to-frequency conversion in the cavity resulting from the measured conversion coefficient of $9.1 \mathrm{~Hz} / \mu \mathrm{W}$. The measured contribution of the power fluctuations of the stabilized laser to the residual frequency stability was observed to be negligible.

\section{Microwave generation}

\subsection{Experimental set-up}

The SESAM-mode-locked ERGO comb with an emission spectrum centred at $1,560 \mathrm{~nm}$ has been used to transfer the relative frequency stability of the PW-ECL locked to the ULE cavity to a microwave signal. The comb was locked to the ultra-stable laser and a harmonic of the repetition rate (at around $10 \mathrm{GHz}$ in the present case) constitutes the microwave output signal.

The ERGO laser emits a pulse train at a repetition rate $f_{\text {rep }} \approx 75 \mathrm{MHz}$ with an average output power of $\sim 100 \mathrm{~mW}$. The major part of this output power was used for CEO generation and detection in a standard $f$-to$2 f$ interferometer [33]. The CEO frequency was phaselocked to a 20-MHz external reference from an H-maser by feedback to the current of the pump diode [21]. For microwave generation via frequency division, an optical line of the self-referenced ERGO comb was phase-locked to the ultra-stable reference laser as schematized in the upper frame of Fig. 4. A fraction of the light at the output of the ERGO laser that was not used for CEO detection and stabilization was split into two parts. In a first arm, an optical power of $\sim 10 \mathrm{~mW}$ was sent to a fast photodiode via a 7-m long thermally isolated single-mode fiber for detection of the repetition rate harmonics. In the second arm, the $\sim 15$-nm wide spectrum with $\sim 400 \mu \mathrm{W}$ average power was combined with $\sim 100 \mu \mathrm{W}$ from the ultra-stable PW-ECL in a 50/50 fibered power combiner. The resulting beat-note signal detected in a $2-\mathrm{GHz}$ bandwidth InGaAs photodiode (Thorlabs DET01CFC) was phase-stabilized to a $10-\mathrm{MHz}$ external reference frequency synthesized by a direct digital synthesizer. The phase error signal was obtained in a digital phase detector with a large linear detection range of $\pm 32 \times 2 \pi$ phase difference [34]. The feedback signal was applied to a piezo-electric transducer (PZT) that controls the laser resonator length with a servo
Fig. 3 (Color online) Scheme of the PDH stabilization of the PW-ECL to the ULE cavity. EOM electro-optic modulator, $A O M$ acousto-optic modulator, $P B S$ polarization beamsplitter, L1-L2 mode matching lenses, $L 3$ focusing lens, $P D$ photodiode, $D B M$ doublebalanced mixer, $P I D$ proportional-integral-derivative servo-controller. Blue fibers are polarization-maintaining fibers; yellow fibers are standard single-mode fibers

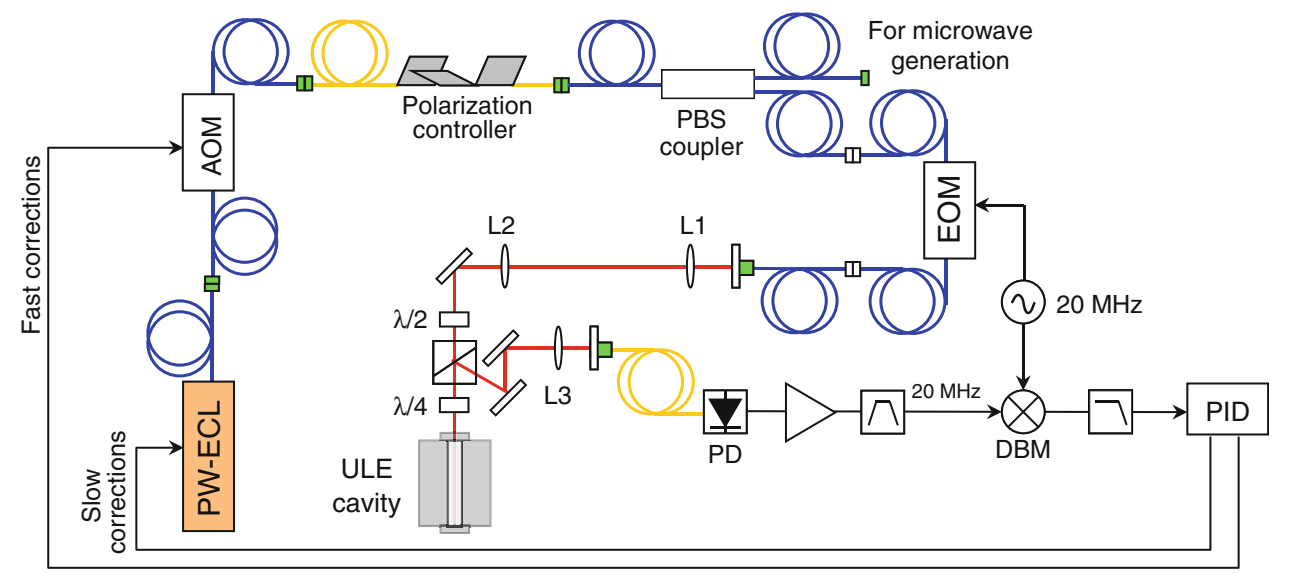




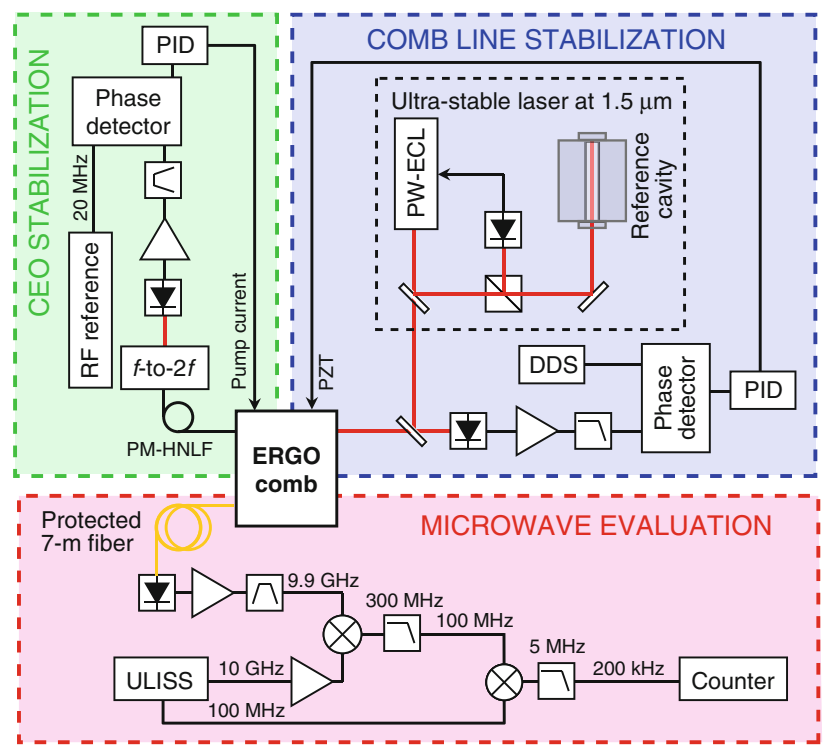

Fig. 4 (Color online) Schematic representation of the microwave generation from the ERGO comb, phase-stabilized to an ultra-stable PW-ECL (upper right frame), and of its evaluation with ULISS (lower frame). PM-HNL polarization-maintaining highly nonlinear fiber (for supercontinuum generation), $D D S$ direct digital synthesizer, $P I D$ proportional-integral-derivative servo-controller

bandwidth in the kilohertz range. Despite this moderate bandwidth, a robust and reliable lock of the comb to the laser was achieved enabling the fractional frequency stability of the laser to be transferred to the comb. A higher feedback bandwidth can be achieved by feedback to the pump current in combination with a subtraction of the CEO beat from the comb-laser heterodyne beat as sometimes implemented with Er:fiber combs [13]. Alternatively, the use of an intra-cavity EOM can be used for further enlargement of the feedback bandwidth [14, 16], but none of these options has been considered here.

With the comb locked to the reference laser, the repetition rate or any of its harmonics constitutes the ultrastable microwave output signal. This was detected using a fast InGaAs pigtailed photodiode (Discovery Semiconductors Inc. DSC30S) with 14-GHz bandwidth. The evaluation of the generated microwave signal was performed by comparison with ULISS [29]. This oscillator, developed at Femto-ST in Besançon, is a transportable version of a cryogenically cooled sapphire oscillator based on the same design as ELISA built for the European Space Agency [35]. It generates a $9.988-\mathrm{GHz}$ frequency reference from a whispering gallery mode in a high-quality factor sapphire resonator cooled down to $6 \mathrm{~K}$ in a pulse-tube cryo-cooler. ULISS offers a relative frequency stability (Allan deviation) better than $3 \times 10^{-15}$ for integration times $\tau$ in the range $1 \mathrm{~s}<\tau<10,000 \mathrm{~s}$ at its fundamental oscillator frequency of $9.988 \mathrm{GHz}$, as well as at several synthesized frequency outputs (e.g., $10 \mathrm{GHz}, 100 \mathrm{MHz}$ or $10 \mathrm{MHz}$ ) with equal or slightly degraded (in the case of $10 \mathrm{MHz}$ ) performances. This makes this oscillator very valuable for the evaluation of microwave signals generated from optical frequency standards.

The frequency comparison has been performed in the $10-\mathrm{GHz}$ range. A component at $9.9 \mathrm{GHz}$ in the detected comb pulse train (corresponding to the 132th harmonic of the comb repetition rate) was band-pass filtered with the use of a narrowband home-made cavity filter, then amplified and frequency down-converted to $\sim 100 \mathrm{MHz}$ by mixing with the synthesized $10-\mathrm{GHz}$ output signal from ULISS. With $\sim 10 \mathrm{~mW}$ incident optical power, the photodiode generates an average photocurrent of $\sim 5 \mathrm{~mA}$ and a microwave signal of about $-34 \mathrm{dBm}$ at $9.9 \mathrm{GHz}$ (before amplification). A second frequency down-conversion was performed by mixing the $\sim 100-\mathrm{MHz}$ signal with the synthesized $100-\mathrm{MHz}$ output signal from ULISS. After low-pass filtering, a signal at around $200 \mathrm{kHz}$ was obtained, which carries the same absolute frequency fluctuations as the 9.9-GHz harmonics of the comb repetition rate, corresponding to an enhancement by a factor of $\sim 5 \times 10^{4}$ in terms of fractional frequency fluctuations. A high precision $\Lambda$-type frequency counter (HP53132A) referenced to an $\mathrm{H}$-maser was used to record the frequency of this down-converted signal with 1-s gate time and the Allan deviation at $1 \mathrm{~s}$ was processed from the recorded frequency series to assess the stability of the generated microwave. It should be noticed that the data obtained with the type of counter used here are not compatible with the standard definition of the Allan deviation [36]. They lead instead to a different representation that is referred to as the triangle variance [37]. The triangle variance shows the same slope with respect to the integration time $\tau$ in the $\log$ $\log$ Allan plot as the modified Allan deviation as first noticed by Rubiola [37] and discussed later in details by Dawkins et al. [38]. It differs from the standard Allan variance in the case of white phase noise. The HP53132A instrument is a modern counter that allows distinguishing the five most common types of noise encountered in oscillators. Depending on the dominant type of noise affecting the analyzed signal, it can lead to an improved fractional frequency stability compared with the standard Allan deviation, especially in presence of white phase noise. For these reasons, the HP53132A counter (as well as the HP53131A from the same family) is quite widely used for the characterization of high stability oscillators [5, 39]. However, one should also point out that this counter cannot be read without dead time between consecutive measurements. This is an issue for the evaluation of oscillators affected by white phase noise or flicker phase noise, for which the correlation between successive frequency measurements is lost in presence of a dead time. Such data can thus not be juxtaposed to calculate an Allan or modified 
Allan deviation for an integration time longer than the gate time without distorting the result [40]. For white phase noise, this leads to a $\tau^{-1 / 2}$ dependence of the Allan deviation instead of a $\tau^{-1}$ dependence obtained with a dead time-free counter. Such a distortion has been reported, e.g., by Bernhardt et al. [41]. For other types of noise (e.g., white frequency noise, flicker frequency noise or random walk frequency noise), the presence of a dead time in the counter measurement has no significant impact on the processed Allan or modified Allan deviation, as successive samples are anyway uncorrelated.

A similar experimental setup was used to generate and evaluate the microwave obtained using a commercial passively mode-locked $\mathrm{Er}^{3+}$ fiber ring frequency comb (FC1500 from MenloSystems) for comparison. This selfreferenced comb was stabilized to the ultra-stable laser using a very similar scheme with feedback applied to the cavity PZT. A frequency down-converted signal at $\sim 188 \mathrm{kHz}$ was generated in a single step by beating the 40th harmonic of the $\sim 250-\mathrm{MHz}$ comb repetition rate with the $9.988-\mathrm{GHz}$ fundamental oscillator frequency from ULISS. The phase noise PSD of the microwave generated from the two combs was also measured by analyzing the phase noise of the same frequency down-converted signals using a digital frequency discriminator (HF2PLL from Zurich Instrument, Switzerland [31]) and an FFT spectrum analyzer.

\subsection{Experimental results}

A similar frequency instability of $5 \times 10^{-15}$ at $1 \mathrm{~s}$ (triangle deviation) was obtained for each comb in comparison with ULISS. Frequency stability for longer integration times has not been processed because of the presence of a dead time in the used counter data that can lead to a distortion of the calculated triangle variance depending on the type of noise as discussed in Sect. 3.1. A linear drift of $74 \mathrm{mHz} / \mathrm{s}$ attributed to the isothermal creep of the ULE cavity [42] was observed in the recorded counter data. The present limitation in the measured frequency stability has not yet been identified, but it is believed to originate from the ultra-stable laser itself and not from the frequency division process in the combs. Some possible causes for this excess noise have been carefully investigated, such as (1) power-to-phase noise conversion in the photo-detection of the comb pulse train, (2) noise induced in the optical fibers used to transfer the laser light to the comb and the comb light to the detection photodiode, and (3) power-tofrequency noise in the reference ULE cavity, but none of them was identified to be responsible for the observed instability.

Due to this excess noise, the fundamental thermal noise limit of our all-ULE cavity (estimated to correspond to an
Allan deviation of $7 \times 10^{-16}$ based on the calculation of Numata et al. [43] and on the analysis of Webster et al. [42] applied to our cavity and incident beam geometries) has not yet been reached. In these conditions, the ERGO comb proved to be as good as the commercial Er:fiber comb in the optical-to-microwave frequency division process.

In Fig. 5, the phase noise of the microwave generated from the ERGO comb is shown to be up to $20 \mathrm{~dB}$ lower compared with the commercial Er:fiber comb at $1-100 \mathrm{kHz}$ offset from the $10-\mathrm{GHz}$ carrier frequency. In particular, the CEO servo bump is barely distinguishable in the microwave signal generated from the ERGO comb, whereas it has a significant contribution in the signal produced by the commercial Er:fiber comb. This is a consequence of the low-noise properties of the CEO in the ERGO comb and of the resulting tight lock that is achieved with a moderate feedback bandwidth [21]. However, one must acknowledge that the Er:fiber comb used here for comparison is not at the state-of-the-art in terms of noise performance. Much lower noise of both tightly locked CEO beat and $10-\mathrm{GHz}$ microwave signal have been demonstrated by Quinlan et al. using a nonlinear polarization rotation mode-locked Er:fiber laser with an intracavity EOM [14]. In that case, the phase noise of the generated $10-\mathrm{GHz}$ microwave signal was $10-15 \mathrm{~dB}$ lower than the results of the ERGO comb shown in Fig. 5 and the contribution of the CEO servo bump to the microwave phase noise was only $\sim-140 \mathrm{dBc} / \mathrm{Hz}$ (at $\sim 25 \mathrm{kHz}$ offset).

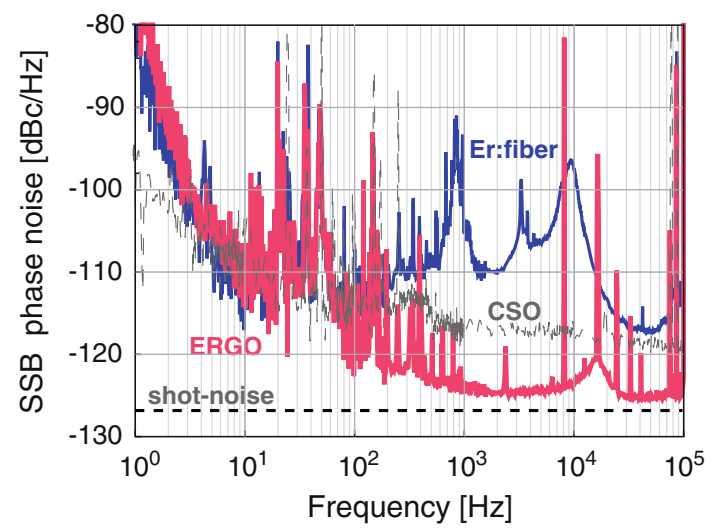

Fig. 5 (Color online) Single-sideband (SSB) phase noise PSD of the $10-\mathrm{GHz}$ microwave generated with the ERGO comb and with a commercial Er:fiber comb for comparison, both evaluated against ULISS. The dashed gray curve shows the phase noise of a CSO obtained in an early comparison between two similar oscillators. Since this time, the short-term stability of ULISS has been improved so that its phase noise in our experimental conditions is very likely lower. The horizontal dashed line indicates the estimated shot-noise floor in the photo-detection of the ERGO comb pulse train. With an incident optical power of $\sim 10 \mathrm{~mW}$, the generated photocurrent was $\sim 5 \mathrm{~mA}$ and the amplitude of the microwave signal at $9.9 \mathrm{GHz}$ was about $-34 \mathrm{dBm}$ 
The phase noise observed in Fig. 5 at an offset frequency ranging from 5 to $100 \mathrm{~Hz}$ from the $10-\mathrm{GHz}$ carrier might result from the noise of ULISS used in the characterization of the microwave generated from each comb, arising in particular from the high servo gain of the Pound stabilization in the sapphire oscillator loop. Unfortunately, the phase noise of ULISS as used in our experiments is not precisely known. The only available data concerns an early comparison made at Femto-ST between two CSOs with a similar resonator, one cryo-cooled and the other operated with liquid Helium. This measurement is shown as a dashed line in Fig. 5, where $3 \mathrm{~dB}$ have been subtracted as generally adopted when comparing two equivalent oscillators. Since this measurement, the short-term frequency stability of ULISS has been improved, but no more recent measurement of the phase noise spectrum has been available. Therefore, we used this early measurement as a first estimation of the phase noise of ULISS, but the actual phase noise is very likely lower than indicated in Fig. 5. This explains why the phase noise measured for the microwave generated with the ERGO comb appears lower than the estimated CSO noise floor at offset frequencies between $100 \mathrm{~Hz}$ and $100 \mathrm{kHz}$ from the $10-\mathrm{GHz}$ carrier. At this point, one cannot conclude whether the measured phase noise was limited by the ERGO comb microwave or by ULISS at offset frequencies above a few hertz. Above $1 \mathrm{kHz}$ offset frequency, the phase noise might be limited by the photodetector shot-noise as indicated by the estimated value of about $-127 \mathrm{dBc} / \mathrm{Hz}$ (horizontal dashed line in Fig. 5). However, the phase noise closer to the carrier is probably slightly higher in the optically generated microwave than in ULISS, which explains the higher fractional frequency instability observed at $1 \mathrm{~s}$ integration time in comparison with ULISS, even if the measurement shown in Fig. 5 is limited by the instrumental noise floor at low frequency.

\section{Discussion and conclusion}

A commercial PW-ECL (ORION module from Redfern Integrated Optics Inc.) has been used to realize an ultrastable laser based on PDH stabilization to an ultra-highfinesse ULE cavity. An AOM was used to correct for fast frequency fluctuations to overcome the limited control bandwidth of the laser injection current. The fractional frequency stability of the cavity-stabilized laser has been transferred to the microwave domain using the SESAMmode-locked ERGO frequency comb, as well as using a commercial Er:fiber comb for comparison. Both combs were phase-locked to the ultra-stable laser by feedback to an intra-cavity PZT with a similar bandwidth. The generated microwave signals have been evaluated against the transportable cryo-cooled sapphire oscillator ULISS, developed at Femto-ST in Besançon and moved to Neuchâtel for a measurement campaign. This transportable sapphire oscillator constitutes a very useful tool for the evaluation of such a stable laser owing to its remarkable short-term frequency stability in the low $10^{-15}$ at timescales up to $10,000 \mathrm{~s}$.

The achieved relative frequency stability of the microwave signal generated from both frequency combs amounts to $5 \times 10^{-15}$ at $1 \mathrm{~s}$ (triangle deviation) and is presently limited by excess noise of the cavity-stabilized laser, whose origin has to be further investigated. With the Er:fiber comb, this is achieved despite the 20-fold higher frequency instability of the CEO beat, whose contribution to the fractional frequency instability of an optical line was determined to be at the level of $2 \times 10^{-14} \tau^{-1}$ [21]. The much better stability achieved for the microwave generated from this comb is a result of the coupling between the two servo-loops in this comb, which was described in detail in the case of the comb stabilized to an RF reference [44], but a similar effect occurs when the comb is locked to an optical reference as considered here.

These results constitute, to the best of our knowledge, the first-reported ultra-stable microwave generation using a DPSSL frequency comb in the $1.5-\mu \mathrm{m}$ spectral range. In comparison with the prior work of Meyer et al. [22] who showed the first microwave generated from a DPSSL comb using an amplified and temporally compressed Yb:KYW femtosecond laser at $1 \mu \mathrm{m}$, the phase noise of the microwave signal obtained here with our ERGO comb is more than $25 \mathrm{~dB}$ better at $10 \mathrm{~Hz}$ offset frequency when scaled to the same carrier frequency of $2 \mathrm{GHz}$ as used by Meyer. However, our results show about $15 \mathrm{~dB}$ higher noise than the new improved results at $10 \mathrm{GHz}$ very recently published by Meyer et al. [23] during the revision of the present paper. Nevertheless, the ERGO comb used here offers the advantage to avoid any pulse compression or amplification as required with the $1-\mu \mathrm{m}$ Yb:KYW DPSSL used by Meyer. Furthermore, it operates at the optical telecommunication wavelength of $1.55 \mu \mathrm{m}$ so that it constitutes an attractive candidate for the simultaneous dissemination of low-noise optical and microwave signals through optical fibers [45]. The phase noise obtained with our DPSSL was also observed to be up to $20 \mathrm{~dB}$ lower than obtained from a commercial Er:fiber comb in a large Fourier frequency range of $100 \mathrm{~Hz}-100 \mathrm{kHz}$ offset from a $10-\mathrm{GHz}$ carrier in similar stabilization conditions. This is a consequence of the much lower phase noise of the CEO beat in the ERGO comb, for which a tight lock is achieved with a moderate feedback bandwidth to the pump current. This makes the CEO servo bump barely distinguishable in the microwave signal generated from the ERGO comb, whereas it has a significant contribution in the Er:fiber 
comb. The microwave phase noise obtained using the ERGO comb is still 10-25 dB higher than state-of-the-art results reported using Ti:Sa combs [12] and $15 \mathrm{~dB}$ higher than achieved with a state-of-the-art Er:fiber comb [14]. However, it is comparable or even $3-5 \mathrm{~dB}$ lower than the phase noise reported for a microwave signal generated from a commercial Er:fiber comb by Millo et al. [3] using a similar stabilization scheme, i.e., without the implementation of pump current-based power lock [15] or an intracavity EOM [14]. The achieved results show that ultrafast DPSSL optical frequency combs stabilized onto an ultrastable reference lasers are attractive candidates for lownoise ultra-stable microwave generation, providing a comparable phase noise level as CSOs and an improved phase noise as compared to a commercial Er:fiber comb stabilized in similar conditions.

Acknowledgments The work at Laboratoire Temps-Fréquence was financed by the Swiss National Science Foundation (SNSF) and by the Swiss Confederation Program Nano-Tera.ch, scientifically evaluated by SNSF. Authors from Femto-ST are grateful to the Fond Européen de Dévelopment Régional (FEDER), the Regional Council of Franche-Comté and OSEO for their support to the ULISS project. We thank L.-G. Bernier (METAS) for fruitful discussions about the impact of frequency counters on stability measurements.

\section{References}

1. M. Armano et al., LISA Pathfinder: the experiment and the route to LISA. Class. Quantum Gravity 26(9), 094001 (2009)

2. S. Weyers, B. Lipphardt, H. Schnatz, Reaching the quantum limit in a fountain clock using a microwave oscillator phase locked to an ultrastable laser. Phys. Rev. A 79, 031803 (2009)

3. J. Millo, M. Abgrall, M. Lours, E.M.L. English, H. Jiang, J. Guéna, A. Clairon, S. Bize, Y. Le Coq, G. Santarelli, M.E. Tobar, Ultra-low noise microwave generation with fiber-based optical frequency comb and application to atomic fountain clock. Appl. Phys. Lett. 94, 141105 (2009)

4. J.G. Hartnett, N.R. Nand, E.N. Ivanov, G. Santarelli, Ultra-stable very-low phase-noise signal source for very long baseline interferometry using a cryocooled sapphire oscillator. IEEE Trans. Microw. Theory Tech. 59, 2978 (2011)

5. J.G. Hartnett, N.R. Nand, C. Lu, Ultra-low-phase-noise cryocooled microwave dielectric-sapphire-resonator oscillators. Appl. Phys. Lett. 100, 183501 (2012)

6. T.W. Hänsch, Nobel lecture: passion for precision. Rev. Mod. Phys. 78, 1297-1309 (2006)

7. J. Ye, J.L. Hall, S.A. Diddams, Precision phase control of an ultrawide-bandwidth femtosecond laser: a network of ultrastable frequency marks across the visible spectrum. Opt. Lett. 25(22), 1675-1677 (2000)

8. B.C. Young, F.C. Cruz, W.M. Itano, J.C. Bergquist, Visible lasers with subhertz linewidths. Phys. Rev. Lett. 82(19), 3799-3802 (1999)

9. J. Millo, D.Y. Magalhães, C. Mandasche, Y. Le Coq, E.M.L. English, P.G. Westergaard, J. Lodewyck, S. Bize, P. Lemonde, G. Santarelli, Ultra-stable lasers based on vibration insensitive cavities. Phys. Rev. A 77(3), 033847 (2008)

10. A.D. Ludlow, X. Huang, M. Notcutt, T. Zanon-Willette, S.M. Foreman, M.M. Boyd, S. Blatt, J. Ye, Compact, thermal-noise- limited optical cavity for diode laser stabilization at $1 \times 10^{-15}$. Opt. Lett. 32(6), 641-643 (2007)

11. T. Kessler, C. Hagemann, C. Grebing, T. Legero, U. Sterr, F. Riehle, M.J. Martin, L. Chen, J. Ye, A sub-40-mHz-linewidth laser based on a silicon single-crystal optical cavity. Nat. Photonics 6, 687-692 (2012)

12. T.M. Fortier, M.S. Kirchner, F. Quinlan, J. Taylor, J.C. Bergquist, T. Rosenband, N. Lemke, A. Ludlow, Y. Jiang, C.W. Oates, S.A. Diddams, Generation of ultrastable microwaves via optical frequency division. Nat. Photonics 5, 425-429 (2011)

13. J. Millo, M. Abgrall, M. Lours, E.M.L. English, H. Jiang, J. Guéna, A. Clairon, S. Bize, Y. Le Coq, G. Santarelli, Ultra-low noise microwave generation with fiber-based optical frequency comb and application to atomic fountain clock. Appl. Phys. Lett. 94, 141105 (2009)

14. F. Quinlan, T.M. Fortier, M.S. Kirchner, J.A. Taylor, M.J. Thorpe, N. Lemke, A.D. Ludlow, Y. Jiang, S.A. Diddams, Ultralow phase noise microwave generation with an Er:fiberbased optical frequency divider. Opt. Lett. 36, 3260-3262 (2011)

15. W. Zhang, Z. Xu, M. Lours, R. Boudot, Z. Kersal, A.N. Luiten, Y. Le Coq, G. Santarelli, Advanced noise reduction techniques for ultra-low noise optical-to-microwave division with femtosecond fiber combs. IEEE Trans. Ultrason. Ferroelectr. Freq. Control 58(5), 900-908 (2011)

16. D.D. Hudson, K.W. Holman, R.J. Jones, S.T. Cundiff, J. Ye, D.J. Jones, Mode-locked fiber laser frequency-controlled with an intracavity electro-optic modulator. Opt. Lett. 30(21), 2948-2950 (2005)

17. T.K. Kim, Y. Song, K. Jung, C. Kim, H. Kim, C.H. Nam, J. Kim, Sub-100-as timing jitter optical pulse trains from mode-locked Er-fiber lasers. Opt. Lett. 36, 4443-4445 (2011)

18. S.A. Diddams, M. Kirchner, T. Fortier, D. Braje, A.M. Weiner, L. Hollberg, Improved signal-to-noise ratio of $10 \mathrm{GHz}$ microwave signals generated with a mode-filtered femtosecond laser frequency comb. Opt. Express 17, 3331-3340 (2009)

19. A. Haboucha, W. Zhang, T. Li, M. Lours, A.N. Luiten, Y. Le Coq, G. Santarelli, Optical-fiber pulse rate multiplier for ultralow phasenoise signal generation. Opt. Lett. 36(18), 3654-3656 (2011)

20. H. Jiang, J. Taylor, F. Quinlan, T. Fortier, S.A. Diddams, Noise floor reduction of an Er:fiber laser-based photonic microwave generator. IEEE Photonics J. 3(6), 1004-1012 (2011)

21. S. Schilt, N. Bucalovic, V. Dolgovskiy, C. Schori, M.C. Stumpf, G. Di Domenico, S. Pekarek, A.E.H. Oehler, T. Südmeyer, U. Keller, P. Thomann, Fully stabilized optical frequency comb with sub-radian CEO phase noise from a SESAM modelocked $1.5-\mu \mathrm{m}$ solid-state laser. Opt. Express 19, 24171 (2011)

22. S.A. Meyer, S. Lecomte, J. Taylor, S.A. Diddams, Low-noise microwave signals from a frequency-stabilized Yb:tungstate optical frequency comb, in CLEO/Europe and EQEC 2009 Conference Digest, (Optical Society of America, 2009), paper PDA_4

23. S.A. Meyer, T.M. Fortier, S. Lecomte, S.A. Diddams, A frequency-stabilized Yb:KYW femtosecond laser frequency comb and its application to low-phase-noise microwave generation. Appl. Phys. B (on-line), doi:10.1007/s00340-013-5439-9

24. R.W.P. Drever, J.L. Hall, F.V. Kowalski, J. Hough, G.M. Ford, A.J. Munley, H. Ward, Laser phase and frequency stabilization using an optical resonator. Appl. Phys. B. 31, 97-105 (1983)

25. M. Alalusi, P. Brasil, S. Lee, P. Mols, L. Stolpner, A. Mehnert, S. $\mathrm{Li}$, Low noise planar external cavity laser for interferometric fiber optic sensors. Proc. SPIE 7316, 73160X (2009)

26. K. Numata, J. Camp, M.A. Krainak, L. Stolpner, Performance of planar-waveguide external cavity laser for precision measurements. Opt. Express 18(22), 22781 (2010)

27. C. Clivati, A. Mura, D. Calonico, F. Levi, G.A. Costanzo, C.E. Calosso, A. Godone, Planar-waveguide external cavity laser 
stabilization for an optical link with $10^{-19}$ frequency stability. IEEE Trans. Ultrason. Ferroelectr. Freq. Control 58(12), 2582-2587 (2011)

28. B. Argence, E. Prevost, T. Lévèque, R. Le Goff, S. Bize, P. Lemonde, G. Santarelli, Prototype of an ultra-stable optical cavity for space applications. Opt. Express 20, 25409-25420 (2012)

29. V. Giordano, S. Grop, B. Dubois, P.-Y. Bourgeois, Y. Kersalé, G. Haye, V. Dolgovskiy, N. Bucalovic, G. Di Domenico, S. Schilt, J. Chauvin, D. Valat, E. Rubiola, New-generation cryogenic sapphire microwave oscillators for space, metrology and scientific applications. Rev. Sci. Instrum. 83(8), 085113 (2012)

30. V. Dolgovskiy, S. Schilt, G. Di Domenico, N. Bucalovic, C. Schori, P. Thomann, $1.5-\mu \mathrm{m}$ cavity-stabilized laser for ultra-stable microwave generation, in Proc. IFCS\&EFTF Joint Conference, San Francisco, USA, May 2-5, 2011

31. S. Schilt, N. Bucalovic, L. Tombez, V. Dolgovskiy, C. Schori, G. Di Domenico, M. Zaffalon, P. Thomann, Frequency discriminators for the characterization of narrow-spectrum heterodyne beat signals: application to the measurement of a sub-hertz carrierenvelope-offset beat in an optical frequency comb. Rev. Sci. Instrum. 82(12), 123116 (2011)

32. G. Di Domenico, S. Schilt, P. Thomann, Simple approach to the relation between laser frequency noise and laser lineshape. Appl. Opt. 49(25), 4801-4807 (2010)

33. H.R. Telle, G. Steinmeyer, A.E. Dunlop, J. Stenger, D.H. Sutter, U. Keller, Carrier-envelope offset phase control: a novel concept for absolute optical frequency measurement and ultrashort pulse generation. Appl. Phys. B 69, 327-332 (1999)

34. M. Prevedelli, T. Freegarde, T.W. Hänsch, Phase locking of grating-tuned diode lasers. Appl. Phys. B 60, S241 (1995)

35. S. Grop, P.Y. Bourgeois, N. Bazin, Y. Kersalé, E. Rubiola, C. Langham, M. Oxborrow, D. Clapton, S. Walker, J. De Vicente, V. Giordano, ELISA: a cryocooled $10 \mathrm{GHz}$ oscillator with $10^{-15}$ frequency stability. Rev. Sci. Instrum. 81, 025102 (2010)
36. D.W. Allan, Statistics of atomic frequency standards. Proc. IEEE 54, 221-230 (1966)

37. E. Rubiola, On the measurement of frequency and its sample variance with high-resolution counters. Rev. Sci. Instrum. 76, 054703 (2005)

38. S.T. Dawkins, J.J. McFerran, A.N. Luiten, Considerations on the measurement of the stability of oscillators with frequency counters. IEEE Trans. Ultrason. Ferroelectr. Freq. Control 54(5), 918-925 (2007)

39. R. Bara, J.-M. Le Floch, M.E. Tobar, P.L. Stanwix, S.R. Parker, J.G. Hartnett, E.N. Ivanov, Generation of $103.75 \mathrm{GHz}$ CW source with $5.10^{-16}$ frequency instability using cryogenic sapphire oscillators. IEEE Micrw. Wirel. Compon. Lett. 22(2), 85-87 (2012)

40. P. Lesage, Characterization of frequency stability: bias due to the juxtaposition of time-interval measurements. IEEE Trans. Instrum. Meas. 32(1), 204-207 (1983)

41. B. Bernhardt, T.W. Hänsch, R. Holzwarth, Implementation and characterization of a stable optical frequency distribution system. Opt. Express 17(19), 16849-16860 (2009)

42. S.A. Webster, M. Oxborrow, S. Pugla, J. Millo, P. Gill, Thermalnoise-limited optical cavity. Phys. Rev. A 77(3), 033847 (2008)

43. K. Numata, A. Kemery, J. Camp, Thermal-noise limit in the frequency stabilization of lasers with rigid cavities. Phys. Rev. Lett. 93, 250602 (2004)

44. V. Dolgovskiy, N. Bucalovic, P. Thomann, C. Schori, G. Di Domenico, S. Schilt, Cross-influence between the two servoloops of a fully-stabilized Er:fiber optical frequency comb. J. Opt. Soc. Am. B 29(10), 2944-2957 (2012)

45. G. Marra, R. Slavík, H.S. Margolis, S.N. Lea, P. Petropoulos, D.J. Richardson, P. Gill, High-resolution microwave frequency transfer over an 86-km-long optical fiber network using a modelocked laser. Opt. Lett. 36(4), 511-513 (2011) 